\title{
Induction-heated nanoimprint on soda-lime glass using sapphire molds
}

\author{
Jingxuan Cai and Shijie Li \\ Department of Mechanical Engineering, The University of Hong Kong, Pokfulam 999077, Hong Kong, \\ China and HKU-Shenzhen Institute of Research and Innovation (HKU-SIRI), Shenzhen 518000, China \\ Xu Guo and Haixong Ge \\ Department of Materials Science and Engineering, Nanjing University, Nanjing 210093, China \\ Wen-Di Li ${ }^{\text {a) }}$ \\ Department of Mechanical Engineering, The University of Hong Kong, Pokfulam 999077, Hong Kong, \\ China and HKU-Shenzhen Institute of Research and Innovation (HKU-SIRI), Shenzhen 518000, China
}

(Received 26 June 2016; accepted 17 October 2016; published 2 November 2016)

\begin{abstract}
The authors developed an induction-heated high-temperature thermal nanoimprint process to directly transfer nanoscale features on sapphire molds to soda-lime glass substrates. Nanoholes of a 650-nm-period hexagonal lattice were directly imprinted in soda-lime glass using the inductionheated thermal nanoimprint configuration as an experimental demonstration. The morphology of nanostructures imprinted on glass substrates at different imprinting process parameters was characterized by scanning electron microscopy and atomic force microscopy and further numerically investigated using a finite element method. The soda-lime glass substrates imprinted with nanohole arrays were then used as secondary templates for further transferring nanostructures onto cyclic olefin copolymer films through another thermal nanoimprint process. The authors also demonstrated surface-enhanced Raman spectroscopy sensing applications on these nanostructured glass substrates after coating them with a thin layer of gold. (C) 2016 American Vacuum Society.
\end{abstract}

[http://dx.doi.org/10.1116/1.4966557]

\section{INTRODUCTION}

Glass is widely used as device substrates or structural materials in many emerging applications in the fields of electronics, ${ }^{1}$ photonics, ${ }^{2}$ microfluidics, ${ }^{3}$ and photovoltaics, ${ }^{4}$ mainly for its high chemical and heat resistance, excellent electrical isolation, high optical transmission in a wide spectral range, low color aberration, and good biocompatibility. Because many of these applications need micro- or nanoscale structures on a substrate surface, development of a suitable process for creating these structures on glass substrates has become increasingly important. Conventional surface structuring of glass can be performed by photolithography and etching ${ }^{5}$ or micromachining. ${ }^{6}$ However, most of these processes are either expensive or in the lack of high resolution for nanostructures.

As a low-cost, high-resolution and large-area mechanical patterning method, nanoimprint lithography (NIL) has received significant development over the past 20 years. ${ }^{7}$ It has the capability to replicate sub- $10 \mathrm{~nm}$ features ${ }^{8}$ with alignment ${ }^{9}$ and even complex three-dimensional structures ${ }^{10}$ by directly deforming the substrate material and is now increasingly used in many emerging optical applications. However, directly nanoimprinting on glass substrates is still challenging because the molding temperature of typical glass substrates is usually above $500^{\circ} \mathrm{C}$, which is much higher than the imprinting temperature for thermoplastic substrates. A reliable compact and fast heating source is desirable for high-temperature nanoimprinting on glass substrates. Moreover, selection of mold materials suitable for imprinting glass under such high temperature and development of a

${ }^{\text {a)} E l e c t r o n i c ~ m a i l: ~ l i w d @ h k u . h k ~}$ reliable and optimized fabrication process are key issues to be resolved. Conventional silicon-based molds used for NIL have strong adhesion to commonly used $\mathrm{SiO}_{2}$-containing glass substrates after high-temperature imprinting, which will increase the risk of mold failure during the nanoimprinting and separation process. Directly imprinting on glass substrates has been reported by researchers. Nanostructures have been patterned on low- $\mathrm{T}_{\mathrm{g}}$ glass using a $\mathrm{Si}_{3} \mathrm{~N}_{4} / \mathrm{SiO}_{2} / \mathrm{Si}$ mold, ${ }^{11}$ where the $\mathrm{Si}_{3} \mathrm{~N}_{4}$ coating is used to physically isolate $\mathrm{SiO}_{2}$-containing glass substrate and $\mathrm{SiO}_{2} / \mathrm{Si}$ mold. Other mold materials, including glassy carbon, ${ }^{6} \mathrm{NiO},{ }^{12}$ and $\mathrm{CVD}$ diamond, ${ }^{13}$ are also employed in the demonstration of nanoimprinting on glass substrates. However, many of the reported studies so far mainly involved rather large structures of a few micrometers or specific low-melting-temperature glass and general modeling for understanding the deformation mechanism during the imprinting process on glass substrates still need further investigation.

In this research, we first developed an induction-heated high-temperature thermal NIL process for replicating nanoscale structures on the most commonly used soda-lime glass using sapphire molds. Induction heating process provides many technological and economic merits in comparison with conventional resistive-heating hot plates for its fast heating rate, high achievable processing temperature, high efficiency, instant controllability, low cost, and compact configuration. ${ }^{14}$ On the other hand, sapphire molds exhibit reliable separation from $\mathrm{SiO}_{2}$-containing glass without additional treatment during experiments. Sapphire molds have previously been used to imprint nanoscale structures on glassy substrates; ${ }^{15-18}$ however, to our best knowledge, high-temperature (e.g., higher than $700^{\circ} \mathrm{C}$ ) thermal nanoimprinting of high-aspect-ratio 
nanostructures on soda-lime glass using sapphire molds have not been reported yet. Here, nanoholes of approximately $200 \mathrm{~nm}$ diameter in a 650-nm pitch hexagonal array were imprinted on soda-lime glass substrates using a sapphire mold carrying the original nanopillar array structure. The effects of imprinting processing parameters, including imprinting temperature and pressure, on the depth of imprinted structure and pattern transfer fidelity, were numerically and experimentally investigated. Then, we used the nanostructured glass substrates as secondary imprinting templates and further transferred the nanostructures into cyclic olefin copolymer (COC) thermoplastic films through another thermal NIL process. Moreover, we also demonstrated potential surface-enhanced Raman sensing (SERS) applications using the nanoimprinted glass substrates after evaporating a thin layer of gold to make them SERS-active.

\section{EXPERIMENT}

Soda-lime glass slides (CAT. 7107P, Fan Yi, China) were cut into $2 \times 2 \mathrm{~cm}$ pieces. These glass pieces were first cleaned with a cotton swab and rinsed thoroughly in deionization (DI) water, and then treated with oxygen plasma (Potentlube, China) for $10 \mathrm{~min}$. The samples were then further cleaned by ultrasonication in isopropanol and DI water for $30 \mathrm{~s}$ before dried in nitrogen. The mold used in this work was a sapphire mold with a $650-\mathrm{nm}$ pitch hexagonal array of nanopillars. The sapphire mold was replicated from a silicon master mold fabricated using interference lithography, e-beam evaporation, and reactive ion etching.

Figure 1 schematizes our work demonstrated in this paper. Initially, circular nanoholes in a 650-nm-pitch hexagonal array were replicated from the sapphire mold to soda-lime glass substrates by a home-built induction-heated thermal nanoimprint

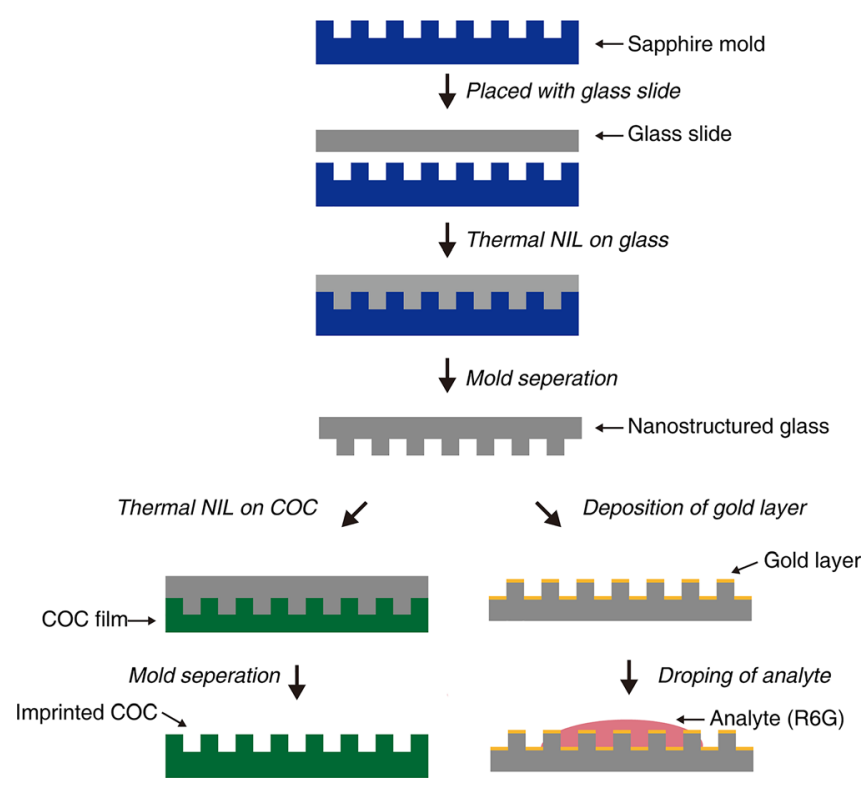

FIG. 1. (Color online) Schematic representation of the induction-heated high-temperature thermal nanoimprint process on soda-lime glass substrates using sapphire molds and applications of nanostructured glass substrates as secondary imprint templates and SERS-active substrates. setup. Then, the nanostructured soda-lime glass substrates were used as templates for two different applications. First, the glass substrates patterned with nanohole arrays were used as secondary molds to reversely replicate nanopillars on $\mathrm{COC}$ films (Grade 8007, TOPAS Advanced Polymers) by a conventional thermal NIL process. Second, in another application demonstration, the nanostructured glass substrates were coated with a thin gold layer to convert them into SERS-active substrates for Rhodamine 6G (R6G) sensing. The details of the experiments are presented in Secs. II A-II C.

\section{A. Induction-heated thermal nanoimprint on soda-lime glass substrates}

A home-built induction-heated thermal nanoimprint setup was used to pattern the soda-lime glass substrates with nanostructures. This setup consisted of a $3.5-\mathrm{cm}$ diameter, $2.5-\mathrm{cm}$ height cylindrical $2 \mathrm{Cr} 13$ ferromagnetic stainless steel press for heat generation by induction, a digital force gauge (Aliyiqi, China) to apply and measure the imprinting pressure, a set of induction heater with a $40-\mathrm{mm}$ diameter brass coil and a HD2440-2 switching power supply (Emerson) to provide $27 \mathrm{~V}$ for the heater.

The imprinting process is schematically described in Figs. 2(a)-2(e). In a typical imprinting process, a cleaned soda-lime glass substrate was first covered by a sapphire mold and supported by another cleaned flat sapphire plate to isolate the glass substrate from the stainless steel press [Fig. 2(a)]. In the following step, the whole stack was placed in the press and heated by a $650 \mathrm{~W}$ induction heater [Fig. 2(b)]. After approximately $2 \mathrm{~min}$, the setup was heated to $820^{\circ} \mathrm{C}\left(90^{\circ} \mathrm{C}\right.$ above the softening point of the soda-lime glass), and set imprinting pressure was applied [Fig. 2(c)]. The induction heater was then powered off, and the system was cooled in the ambient environment. The imprinting pressure was maintained during the whole imprint process until the temperature was lower than the annealing point of the soda-lime glass. Finally, the mold and the substrate can be separated manually after the stack is cooled down to room temperature [Fig. 2(d)]. The general sequence of heating and pressing is summarized in Fig. 2(e). Figure 2(f) shows the thermocouple-measured temperature in the setup as a function of imprinting time during a typical imprinting process. With the compact configuration, it costs approximately $2 \mathrm{~min}$ to cool the system down to the demolding temperature. The cooling process can be accelerated if an external forced cooling device, e.g., a water circulation chiller, is applied. With further optimization, our induction-heated imprint process would be capable of high throughput fabrication of nanostructured soda-lime glass substrates.

In all the experiments presented in this work, sapphire molds were not treated with any antisticking layer, since the antisticking agent cannot withstand such high temperature and sapphire molds showed excellent nature for separation from imprinted glass substrates. The characterizations of samples were performed using Hitachi S-4800, LEO 1530 SEMs, and a Bruker MultiMode-8 AFM. 
(a)

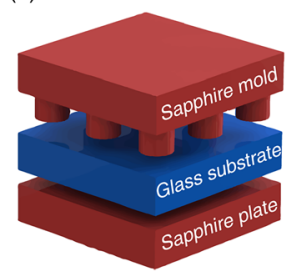

(b)

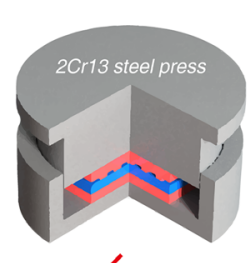

(c)

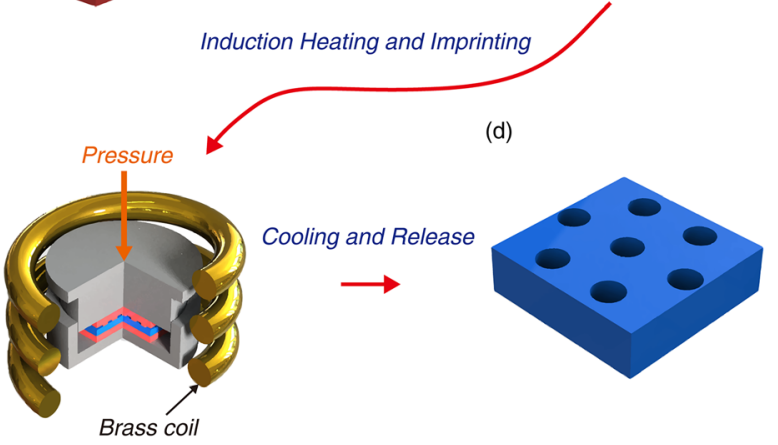

(e)

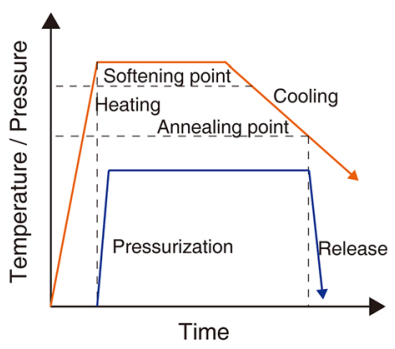

(f)

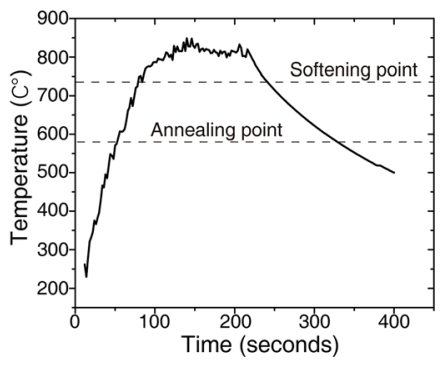

FIG. 2. (Color online) Schematic illustrations of induction-heated nanoimprint process on soda-lime glass substrates. (a) A cleaned glass substrate is covered by a sapphire mold and supported by another flat sapphire plate; (b) the stack is placed in a $2 \mathrm{Cr} 13$ steel press and (c) heated by the induction heater; (d) nanostructures are transferred from the sapphire template onto the glass substrate after cooling and releasing; (e) Sequence of heating and pressing during the imprinting; and (f) plot of measured temperature vs imprint time during a typical imprint process.

\section{B. Pattern transfer on plastic films using directly imprinted glass templates}

Glass substrates patterned with nanohole arrays, fabricated through the aforementioned induction-heated imprint process, were used as secondary templates in subsequent replication process on COC films. ${ }^{19}$ The glass template was positioned on top of a cleaned COC film and then placed in a nanoimprint platform (YPL-NIL-SI400, China). The template/substrate stack was then heated to $120^{\circ} \mathrm{C}$ for 5 min with an imprinting pressure of $0.5 \mathrm{MPa}$ to press the template and the COC film. Afterward, the heated stack was cooled down to the demolding temperature of $50^{\circ} \mathrm{C}$, and the $\mathrm{COC}$ film was peeled off from the glass template after releasing the pressure, leaving nanostructures replicated on the COC film.

\section{Surface-enhanced Raman spectroscopy on directly imprinted soda-lime glass}

Nanostructured glass substrates were first cleaned with DI water and dried in a nitrogen flow. Then, the samples were coated with a 70-nm thickness gold layer by thermal evaporation and were converted into SERS-active substrates. Such

SERS samples feature a vertically coupling structure consisting of disks and holes with small gaps in between and show very high enhancement factor with optimized structural geometry. ${ }^{20}$ An aqueous solution $\left(1 \mu \mathrm{l}, 1.0 \times 10^{-5} \mathrm{M}\right)$ of R6G were dropped on the above gold-coated nanostructured glass substrates and dried under ambient conditions to leave a certain amount of analyte molecules on the SERS substrate surface. Then, the samples were excited by a $785 \mathrm{~nm}$ laser, and the Raman spectra of R6G were recorded using a QE65 Pro Raman spectrometer (Ocean Optics).

\section{RESULTS AND DISCUSSION}

The SEM images of master sapphire molds patterned with 300-nm depth, 650-nm pitch hexagonal nanopillar array and corresponding replicated hexagonal nanohole array on soda-lime glass substrates are shown in Figs. 3(a) and 3(b), respectively. The structure illustrated in Fig. 3(b) was fabricated using an imprinting pressure $\mathrm{P}=9.6 \mathrm{MPa}$. It is evident from the results that the nanoholes in glass are the reversed copies of the nanopillars on the sapphire mold.

Besides these successful replications, a series of inductionheated thermal NIL experiments were carried out using different imprinting pressure to further investigate the effects of the imprinting pressure on the depth of imprinted nanoholes during our imprint process. For each set of experiments, the morphological characterizations were performed by SEM and

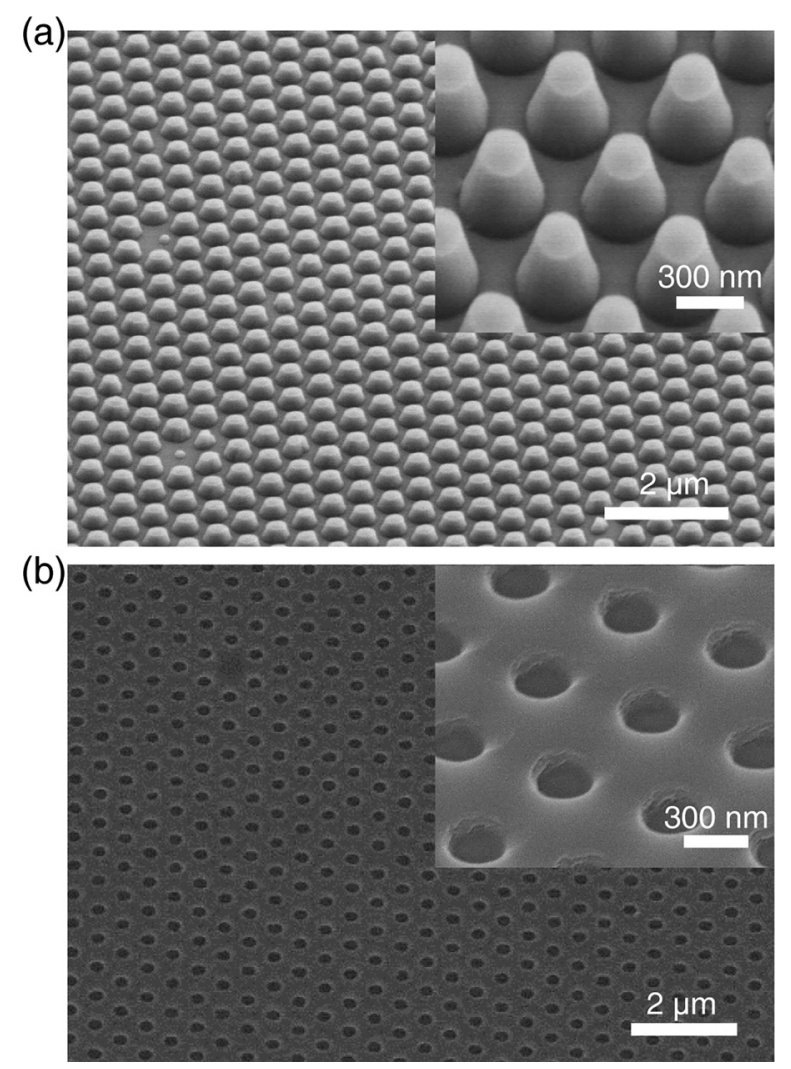

FIG. 3. Morphological characterization of the sapphire mold and imprinted glass substrate by SEM. (a) The 650-nm pitch hexagonal nanopillar array on sapphire molds. (b) The reversely replicated nanohole array on glass substrate fabricated at an imprinting pressure of 9.6 MPa. The SEM images were taken using a $45^{\circ}$ tilted sample holder. 
AFM and the depths of imprinted nanoholes were measured by AFM. Typical morphology and corresponding crosssectional height profiles of the imprinted nanoholes on glass substrates with the imprinting pressures of 5.6, 8.6, and 11.2 MPa were shown in Figs. 4(a)-4(c), respectively. During the experiments, other imprinting parameters remained constant. The results showed that for a given pattern and constant processing parameters, the imprinted depth of nanoholes increases with the imprinting pressure.

We also investigated the effects of the imprinting temperature on the depth of imprinted nanoholes using numerical modeling based on the temperature-dependent viscosity and Young's modulus of soda-lime glass, as shown in Figs. 5(a) and 5(b). ${ }^{21,22}$ Young's modulus describes the elastic deformation of a solid material under tensile or compressive loading, while the viscosity is related to the states of glass at the specific temperature. Glass can be considered as elastic below $\mathrm{T}_{\mathrm{g}}$. When the temperature of glass goes beyond $T_{g}$, the viscosity decreases, so glass shows more viscoelastic characteristics, then glass gradually softens and its Young's modulus decreases; therefore, directly imprinting on glass is possible. Using the temperature-dependent properties of glass, we then implemented numerical simulation to study the effects of imprinting temperature using a finite element method. The systematic illustration of the model was plotted in the inset of Fig. 5(c). The simulation model included a sapphire mold and a soda-lime glass substrate. For simplicity, the mold was assumed as ideally rigid. The glass substrate was considered as a viscoelastic material using the Kelvin-Voigt model, while the ambient temperature was set to be $820^{\circ} \mathrm{C}$ and the viscosity of glass was $5 \times 10^{6} \mathrm{Pas}$. Periodic boundary conditions were applied in the $\mathrm{x}$ - and $\mathrm{y}$-directions. In the Kelvin-Voigt model, ${ }^{23}$ materials can be represented by a purely viscous damp and a purely elastic spring connected in parallel. Comparing with the Generalized Maxwell model, the KelvinVoigt model is more realistic for describing the creeping effect. Although the Kelvin-Voigt model does not describe the relaxation behavior after removal of stress, in the presented cases, the imprinting pressures are always removed when the temperature drops across the annealing temperature. Hence, the relaxation behavior is not important, and the Kelvin-Voigt model is favorable. The time-dependent deformation in the Kelvin-Voigt model upon a sudden constant stress $\sigma_{0}$ is

$$
\varepsilon_{t}=\frac{\sigma_{0}}{E}\left(1-e^{-\lambda t}\right)
$$

where $\lambda$ is the rate of relaxation (a)

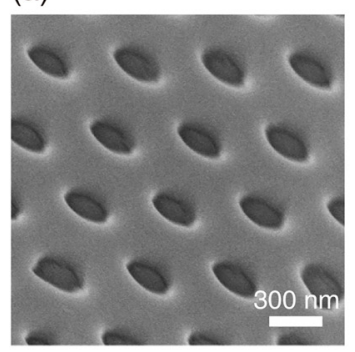

(b)

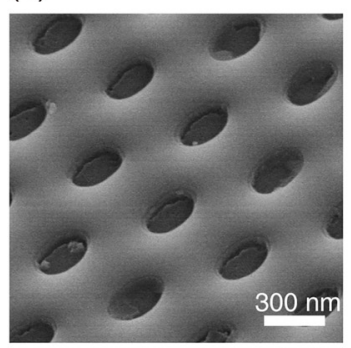

(c)

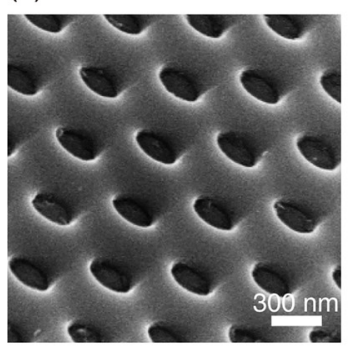

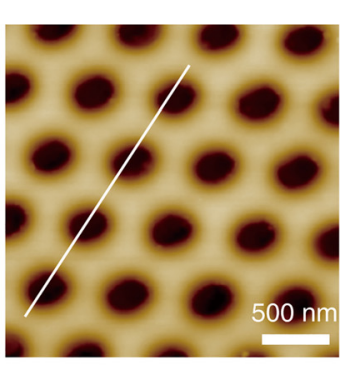

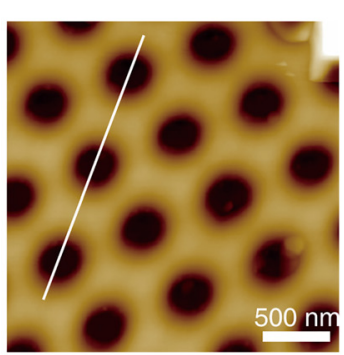

$500 \mathrm{~nm}$

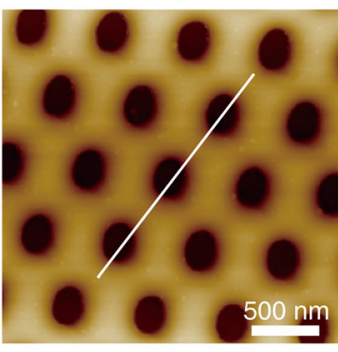

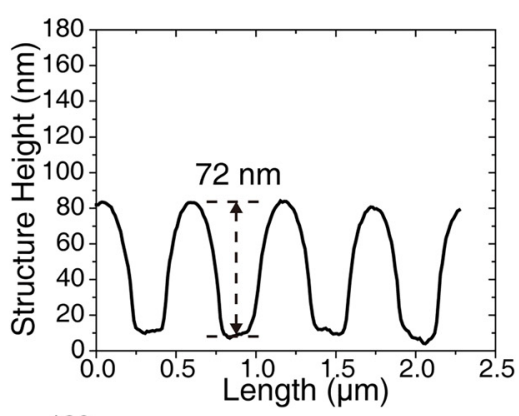
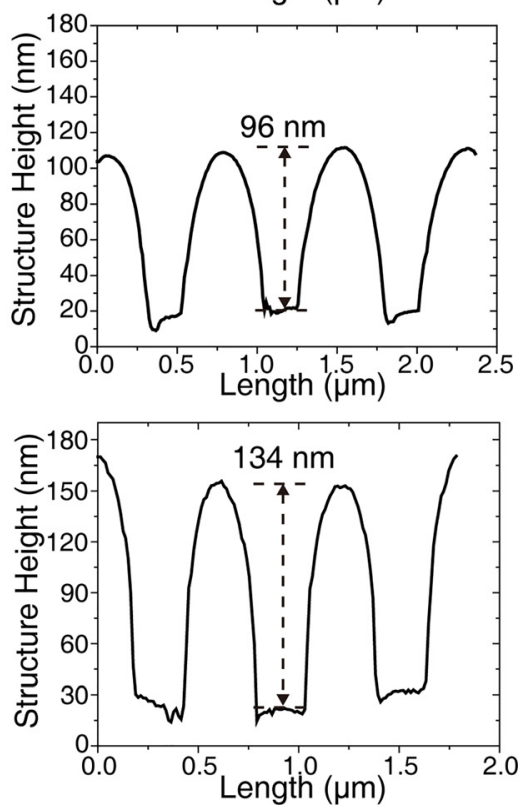

FIG. 4. (Color online) Morphological characterizations of nanostructured soda-lime glass substrates processed by different imprinting pressures by SEM (left), AFM (middle) and corresponding cross-sectional height profiles along the white lines (right). (a) Imprinting pressures $\mathrm{P}=5.6 \mathrm{MPa}$, (b) $\mathrm{P}=8.5 \mathrm{MPa}$, and (c) $\mathrm{P}=11.2 \mathrm{MPa}$. 


$$
\lambda=\frac{E}{\eta} .
$$

In the infinite time limit, the strain approaches a constant

$$
\varepsilon_{\infty}=\frac{\sigma_{0}}{E}
$$

Given the Young's modulus $E=250 \mathrm{kPa}$ and a viscosity $\eta=5 \times 10^{6} \mathrm{Pas}$ at $820^{\circ} \mathrm{C}$ it only costs $0.09 \mathrm{~s}$ to achieve $99 \%$ of $\varepsilon_{\infty}$. The contribution of the creeping effect in lower temperature imprint is even less. Therefore, time in the transient simulation is set to be $30 \mathrm{~s}$ in all of the results.

Figure 5(c) plotted the depth of imprinted nanoholes as a function of imprinting pressure from the numerical modeling and experimental data, respectively. The curve indicated that the imprinted structure depth increases linearly with the imprinting pressure, and the relationship was confirmed by experiment results. Therefore, fulfilling the requirement for transferring higher-aspect-ratio structure on soda-lime glass needs higher imprinting pressure. Meanwhile, nanoholes with different depths for a given nanopillar-patterned template can be obtained simply by changing the imprinting pressure.

The imprint process was further numerically investigated by changing the imprinting temperature, in which imprinting pressure remained constant. The relationship of the imprinting

(a)

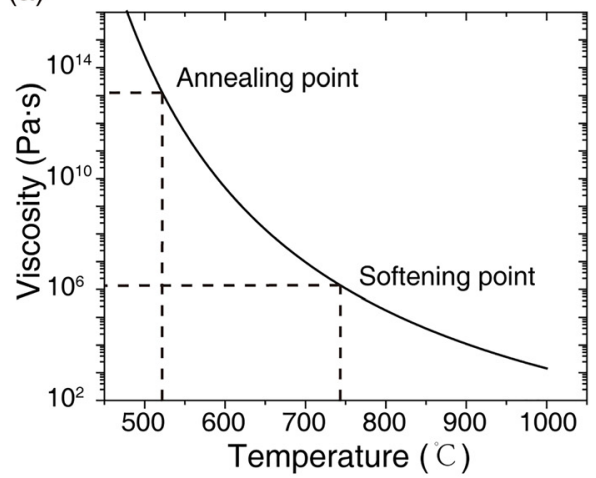

(c)

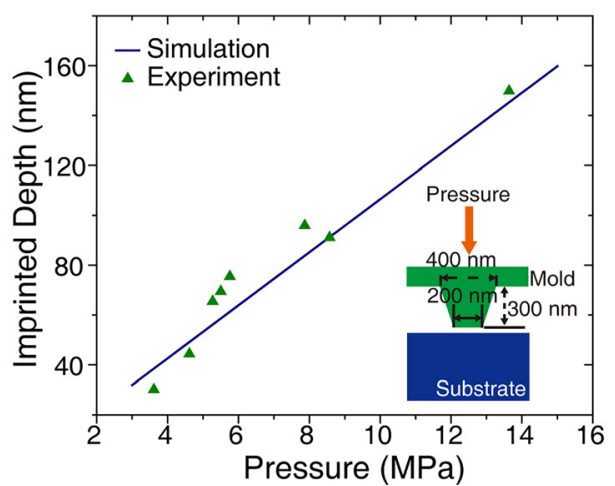

temperature and depth of imprinted nanoholes under two different imprinting pressures, $\mathrm{P}=5 \mathrm{MPa}$ and $\mathrm{P}=10 \mathrm{MPa}$, respectively, was summarized in Fig. 5(d). The curves revealed that the depth of imprinted nanoholes increases dramatically when the imprinting temperature goes across the softening point of glass, which is attributed to the decreasing Young's modulus.

The successfully fabricated hexagonal nanohole arrays on soda-lime glass substrates were used as templates to replicate nanopillar arrays on COC films through another thermal NIL process. The SEM images and the corresponding AFMmeasured cross-sectional height profiles of nanoholes on the glass template and transferred nanopillars on the COC film are shown in Figs. 6(a)-6(d), respectively. Structures on the glass template were reliably replicated on the COC film. We also tested the glass templates for five imprinting cycles to investigate the durability of glass templates, and no noticeable defects, deformation, or collapse of features were found by investigating both the imprinted COC films and the glass templates after the fifth imprinting cycle under optical microscope and SEM, which indicated that the glass templates are reasonably robust and durable.

We also demonstrated the application of using nanostructured glass substrates to fabricate SERS-active substrates. In our experiment, the SERS-active substrate was prepared by evaporating a 70-nm thickness layer of gold onto a soda-lime

(b)

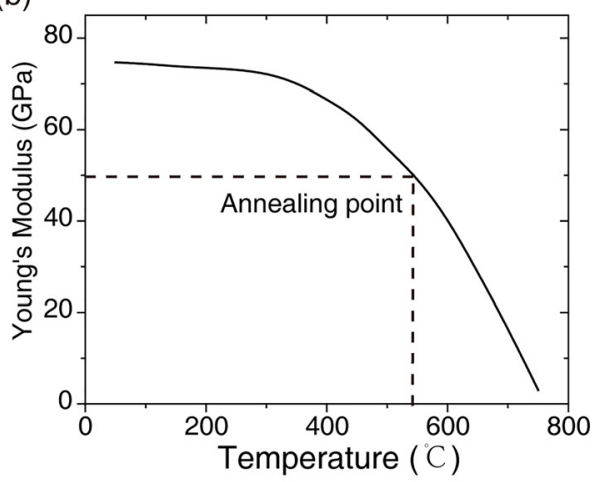

(d)

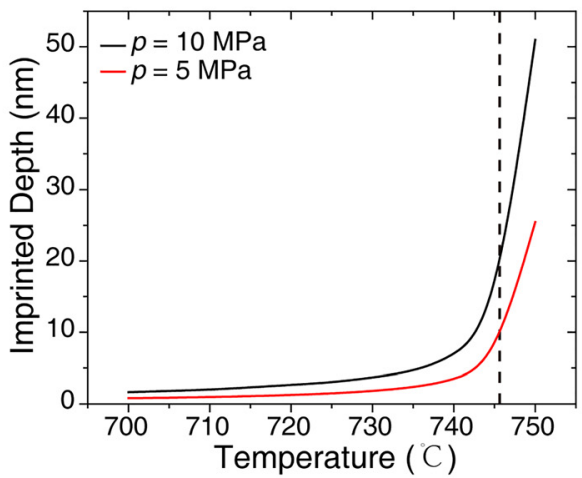

FIG. 5. (Color online) Effects of processing parameters on the depth of imprinted nanoholes. (a) Plot of the viscosity of the soda-lime glass vs temperature, plotted using the VFT equation (Refs. 24-26) and model from Fluegel (Ref. 22). (b) Plot of the Young's modulus of the soda-lime glass as a function of temperature, plotted using the experimental data from McGraw (Ref. 21). (c) Quantitative relationship of the depth of imprinted nanoholes with imprinting pressure from experiments (green triangles) and from numerical simulations (blue line). (Inset) Schematic representation of the simulation structures. (d) Plot of the depth of imprinted nanoholes vs imprinting temperature from simulations under imprinting pressures of 5 and $10 \mathrm{MPa}$, respectively. 
(a)

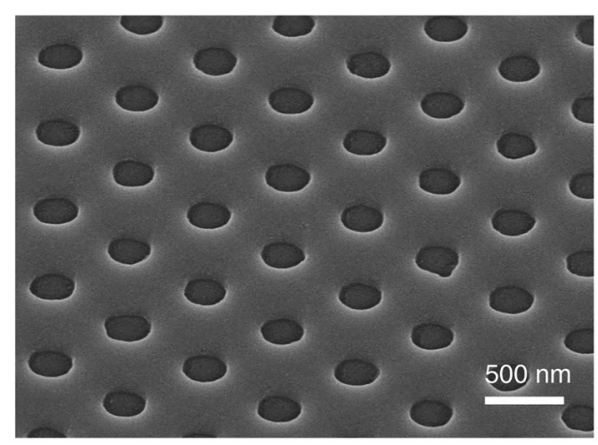

(c)

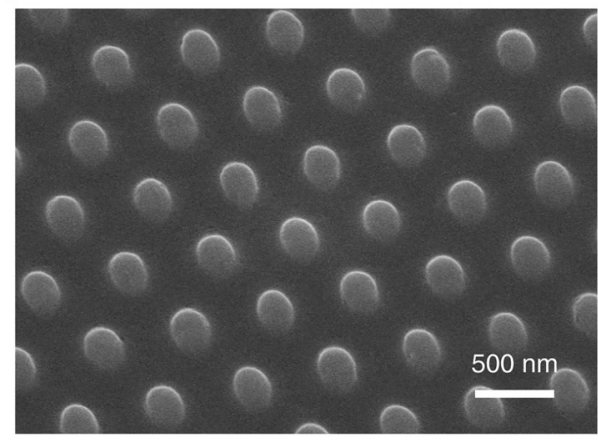

(b)

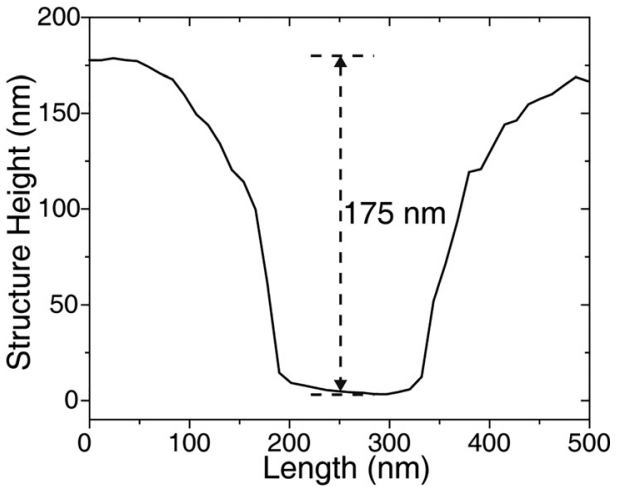

(d)

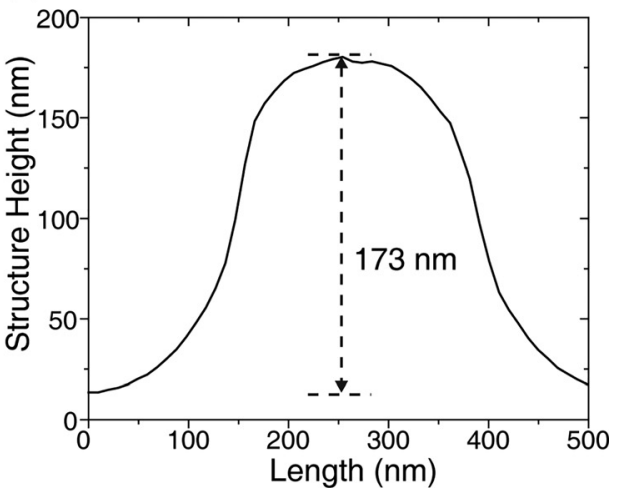

FIG. 6. Pattern transfer on COC films using directly imprinted glass templates. (a) SEM image of 650-nm pitch hexagonal nanohole array on the glass template and (b) corresponding AFM-measured cross-sectional height profile of one hole, the depth of the hole is $175 \mathrm{~nm}$. (c) SEM image of the reversely imprinted nanopillar array on the COC film and (d) corresponding cross-sectional height profile of one pillar, the height of the pillar is $173 \mathrm{~nm}$.

glass substrate imprinted with an $87-\mathrm{nm}$ depth hexagonal nanohole array [Fig. 7(a)]. Then, we dropped $1 \mu \mathrm{l}$ of $1.0 \times 10^{-5} \mathrm{M}$ R6G aqueous solution on the above gold-coated nanostructured substrate and dried it in ambient conditions for $12 \mathrm{~h}$ to leave a certain amount of R6G molecules on the SERS substrate. Afterward, we measured the Raman spectra excited by a $785 \mathrm{~nm}$ laser. As a reference, we also investigated the Raman spectra of the R6G on a flat glass substrate coated with 70-nm-thick gold. Figure 7(b) summarized the Raman spectra measured from these samples. It is extremely difficult to obtain a clear Raman spectrum for such small amount of R6G on the unpatterned glass as the signal is too weak. Compared with the reference sample, considerable enhancement on the R6G signature was obtained on the gold-coated nanostructured substrate for the Raman modes of R6G at 614 , $775,1186,1316$, and $1366 \mathrm{~cm}^{-1}$ excited by a $785 \mathrm{~nm}$ laser.

We then implemented a finite-difference time-domain (FDTD) calculation to further investigate the enhancement behavior of Raman signal in our experiment. For simplicity, we assumed a uniform hexagonal unit cell for the FDTD calculation. The $|\mathrm{E}|^{2}$ distribution of the gold-coated nanostructured glass substrate is shown in Fig. 7(c). The excitation wavelength was chosen at $785 \mathrm{~nm}$ as used in our experiment. It can be observed that enhanced electrical field is localized around the edge, which is believed to be caused by the surface plasmon polariton (SPP) and the coupling between SPP of the top metal nanoholes and localized surface plasmonic resonance of bottom metal nanodisk. Although the structures in this work were limited to 650 -nm pitch hexagonal nanohole arrays due to the availability of the mold we used in the imprint process, with further design and optimization, such metallic nanostructures fabricated on directly imprinted glass substrates can be utilized as high-performance sensors based on surface enhanced spectroscopy techniques.

\section{CONCLUSIONS}

In summary, we developed an efficient method for faithfully and readily transferring nanopatterns onto soda-lime glass substrates by induction-heated high-temperature thermal nanoimprint process using sapphire molds. We experimentally and numerically studied the processing parameters and replication fidelity of transferring nanostructures on soda-lime glass substrates during imprint process. Nanohole arrays were successfully and faithfully transferred on soda-lime glass substrates using 650-nm pitch hexagonal nanopillar array patterned sapphire molds. Our experiment and modeling results confirmed that the depth of imprinted nanoholes increases linearly with imprinting pressure when other processing parameters remain constant during this imprinting process of soda-lime glass substrates. As an application, the imprinted nanostructures on glass substrates were further transferred onto thermoplastic COC films through another conventional thermal NIL process using the nanostructured glass substrates as secondary templates. The replicated reversed nanostructures on the COC film exhibited high fidelity compared with 
(a)

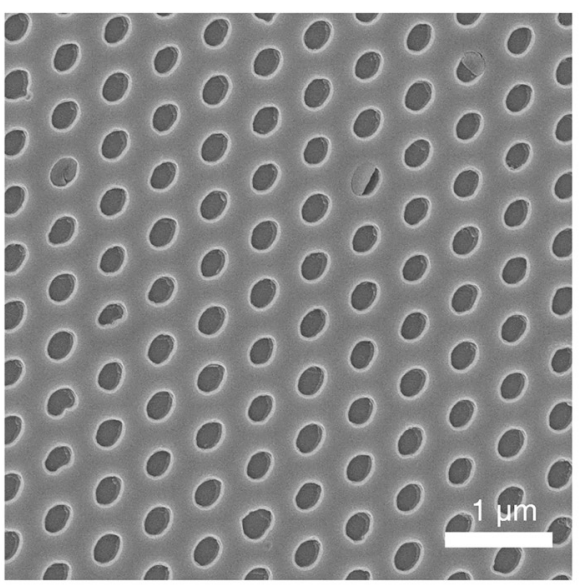

(b)
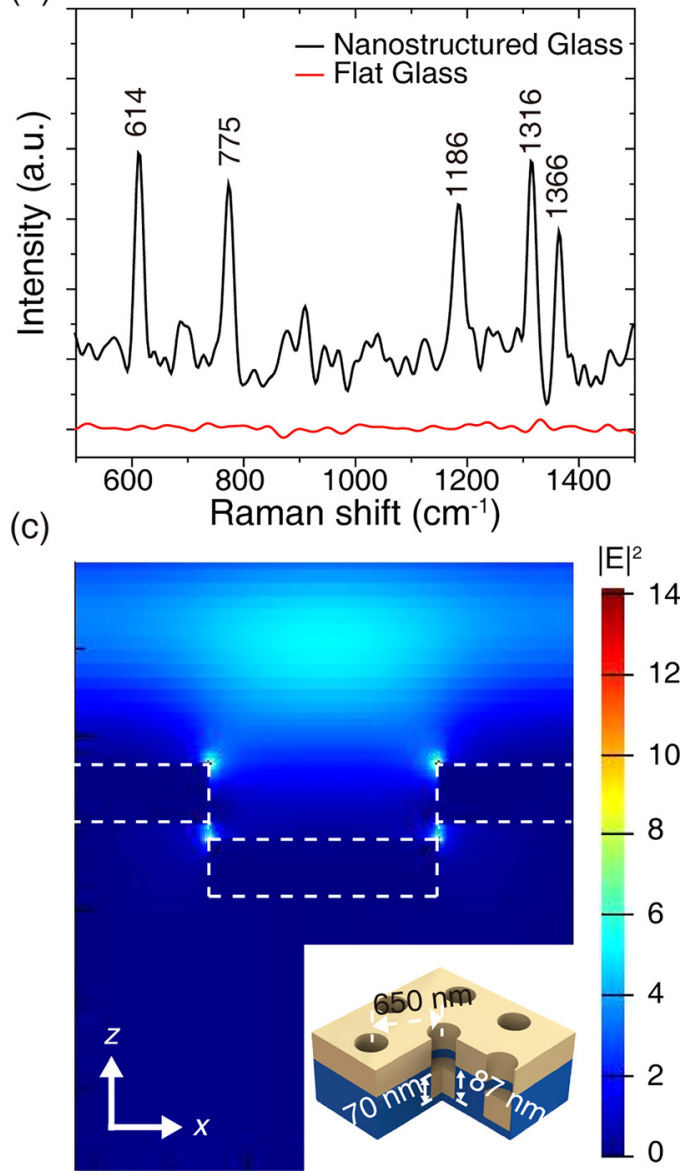

FIG. 7. (Color online) SERS spectroscopy on a directly imprinted glass substrate. (a) SEM image of the imprinted glass substrate before deposition of gold. (b) Raman spectra of R6G obtained using a $785 \mathrm{~nm}$ laser obtained from the gold-coated nanostructured glass substrate (black curve) and reference gold-coated flat glass substrate (red curve). The Raman peaks at 614, $775,1186,1316$, and $1366 \mathrm{~cm}^{-1}$ are characteristic for R6G. (c) Simulated $|\mathrm{E}|^{2}$ at $785 \mathrm{~nm}$ excitation for the substrate structures. (Inset) Schematic illustration of the SERS-active substrate after deposition of gold. The depth of nanoholes is $87 \mathrm{~nm}$, and the thickness of the gold layer is $70 \mathrm{~nm}$. the original patterns on glass templates. Moreover, by depositing a thin layer of Au film on nanostructured soda-lime glass substrates, highly sensitive SERS-active substrates were fabricated and showed good enhancement for R6G sensing.

\section{ACKNOWLEDGMENTS}

This work was partially supported by the National Natural Science Foundation of China (Grant No. 61306123), the Research Grants Council of the Hong Kong Special Administrative Region (Award Nos. 27205515 and 17246116), and the Basic Research Program-General Program (JCYJ20140903112959959) from the Science and Technology Innovation Commission of Shenzhen Municipality.

${ }^{1}$ M. C. McAlpine, R. S. Friedman, S. Jin, K.-H. Lin, W. U. Wang, and C. M. Lieber, Nano Lett. 3, 1531 (2003).

${ }^{2}$ L. Tong, L. Hu, J. Zhang, J. Qiu, Q. Yang, J. Lou, Y. Shen, J. He, and Z. Ye, Opt. Express 14, 82 (2006).

${ }^{3}$ J. Xu, D. Wu, Y. Hanada, C. Chen, S. Z. Wu, Y. Cheng, K. Sugioka, and K. Midorikawa, Lab Chip 13, 4608 (2013).

${ }^{4}$ I. Gordon, F. Dross, V. Depauw, A. Masolin, Y. Qiu, J. Vaes, D. Van Gestel, and J. Poortmans, Sol. Energy Mater. Sol. Cell 95, S2 (2011).

${ }^{5}$ L. Zhang, W. Wang, X. J. Ju, R. Xie, Z. Liu, and L. Y. Chu, RSC Adv. 5, 5638 (2015).

${ }^{6}$ S. Youn, M. Takahashi, H. Goto, and R. Maeda, Microelectron. Eng. 83, 2482 (2006)

${ }^{7}$ S. Y. Chou, P. R. Krauss, and P. J. Renstrom, Appl. Phys. Lett. 67, 3114 (1995).

${ }^{8}$ S. Y. Chou, P. R. Krauss, W. Zhang, L. Guo, and L. Zhuang, J. Vac. Sci. Technol., B 15, 2897 (1997).

${ }^{9}$ W. Wu, R. G. Walmsley, W. D. Li, X. M. Li, and R. S. Williams, Appl. Phys., A 106, 767 (2012).

${ }^{10}$ F. X. Zhang and H. Y. Low, Nanotechnology 17, 1884 (2006).

${ }^{11}$ Y. Hirai, K. Kanakugi, T. Yamaguchi, K. Yao, S. Kitagawa, and Y. Tanaka, Microelectron. Eng. 67-68, 237 (2003).

${ }^{12}$ S. Akiba, W. Hara, T. Watanabe, A. Matsuda, M. Kasahara, and M. Yoshimoto, Appl. Surf. Sci. 253, 4512 (2007).

${ }^{13}$ M. Komori, H. Uchiyama, H. Takebe, T. Kusuura, K. Kobayashi, H. Kuwahara, and T. Tsuchiya, J. Micromech. Microeng. 18, 065013 (2008).

${ }^{14}$ Z. M. Wang, X. G. Yang, Y. H. Wang, and W. L. Yan, IEEE Trans. Magn. 37, 3437 (2001).

${ }^{15}$ M. Komuro, Y. Tokano, J. Taniguchi, T. Kawasaki, I. Miyamoto, and H. Hiroshima, Jpn. J. Appl. Phys., Part 1 41, 4182 (2002).

${ }^{16}$ Y. Akita, T. Watanabe, W. Hara, A. Matsuda, and M. Yoshimoto, Jpn. J. Appl. Phys., Part 2 46, L342 (2007).

${ }^{17}$ M. Yoshimoto, Appl. Phys., A 121, 321 (2015).

${ }^{18}$ O. Suga, M. Yoshimoto, C. Okuyama, A. Matsuda, H. Hiroshima, and M. Takahashi, Jpn. J. Appl. Phys., Part 1 54, 098001 (2015).

${ }^{19}$ A. Khan, S. J. Li, X. Tang, and W. D. Li, J. Vac. Sci. Technol., B 32, 06FI02 (2014).

${ }^{20}$ W. D. Li, F. Ding, J. Hu, and S. Y. Chou, Opt. Express 19, 3925 (2011).

${ }^{21}$ D. McGraw, J. Am. Ceram. Soc. 35, 22 (1952).

${ }^{22}$ A. Fluegel, Glass Technol., Part A 48, 13 (2007).

${ }^{23}$ M. A. Meyers and K. K. Chawla, Mechanical Behavior of Materials. (Cambridge University, Cambridge, 2009).

${ }^{24}$ G. S. Fulcher, J. Am. Ceram. Soc. 75, 1043 (1992).

${ }^{25}$ G. H. W. Z. Tammann and W. Hesse, Z. Anorg. Allg. Chem. 156, 1 (1926).

${ }^{26}$ S. English, J. Soc. Glass Technol. 8, 205 (1924). 